\title{
Hemorragia digestiva e embolização arterial no Brasil: estudo sobre a distribuição e mortalidade
}

\author{
Digestive hemorrhage and arterial embolization in Brazil: a study on distribution and mortality
}

Hemorragia digestiva y embolización arterial en Brasil: estudio de distribución y mortalidad

Shádya Teixeira Amer ${ }^{1}$, Jorge Patrick Silva da Rocha², Apolone da Mota Queiroz ${ }^{1}$, José Wilker Gomes de Castro Júnior ${ }^{2}$, Bárbara Mendonça Paiva Antonio José ${ }^{3}$, Charlon Costa de Oliveira ${ }^{1}$, Lucas Dantas da Silva Mufarrej Hage ${ }^{2}$, Faustino Chaves Calvo ${ }^{3}$, Deivid Ramos dos Santos ${ }^{3 *}$, Renan Kleber Costa Teixeira ${ }^{3}$.

\section{RESUMO}

Objetivos: Analisar a ocorrência da distribuição e mortalidade relacionadas a embolização arterial da hemorragia digestiva realizados no Sistema Único de Saúde. Métodos: Pesquisa quantitativa, retrospectiva e descritiva, realizada a partir da coleta em base de dados disponibilizada pelo Sistema de Informações Hospitalares, sob o código 0406040192, referente ao procedimento de embolização arterial de hemorragia digestiva, no período de 2014 a 2020. Resultados: Foram notificados 1.435 procedimentos de embolização arterial. O Sudeste foi responsável por $48,01 \%$ casos. A Região Norte foi responsável por $0,28 \%$. De todos os procedimentos registrados, 40,63\% são decorrentes do estado de São Paulo, seguidos de Pernambuco $(21,53 \%, n=309)$ e Rio Grande do Sul $(9,69 \%, n=139)$. O Centro-Oeste foi responsável pela maior taxa de mortalidade. Conclusão: Observou-se discrepância da distribuição dos casos por estado e região. Foi identificado completa ausência de registros de mais de 7 estados durante o período estudado, apontando falta dessa modalidade terapêutica e/ou subnotificação e subinformação relacionada ao procedimento analisado.

Palavras-chave: Embolização terapêutica, Hemorragia gastrointestinal, Trato gastrointestinal.

\begin{abstract}
Objective: Objectives: to analyzed the distribution and mortality related to arterial embolization of digestive hemorrhage performed in the Unified Health System. Methods: A quantitative, retrospective and descriptive research, carried out based on the collection in a database made available by the Hospital Information System, under the code 0406040192, referring to the arterial embolization procedure of digestive hemorrhage, in the period from 2014 to 2020. Results: 1,435 arterial embolization procedures were notified. The Southeast was responsible for $48.01 \%$ of cases. The Northern was responsible for $0.28 \%$. Of all registered procedures, $40.63 \%$ is from the state of São Paulo, followed by Pernambuco $(21.53 \%, n=309)$ and Rio Grande do Sul $(9.69 \%, \mathrm{n}=139)$. The Midwest was responsible for the highest mortality rate. Conclusion: There is a discrepancy in the distribution of cases by state and region. A complete absence of records from more than 7 states was identified during the study period, indicating a lack of this therapeutic modality and / or underreporting and under information related to the analyzed procedure.
\end{abstract}

Keywords: Embolization therapeutic, Gastrointestinal hemorrhage, Gastrointestinal tract.

\section{RESUMEN}

Objetivos: Analizar la ocurrencia de distribución y mortalidad relacionada con la embolización arterial de hemorragia digestiva realizada en el Sistema Único de Salud. Métodos: Investigación cuantitativa, retrospectiva y descriptiva, realizada a partir de la recolección en una base de datos proporcionada por el Sistema de Información Hospitalaria, bajo el código 0406040192, referido al procedimiento de embolización arterial por hemorragia digestiva, de 2014 a 2020. Resultados: Se reportaron 1,435 procedimientos de

\footnotetext{
${ }^{1}$ Fundação Hospital de Clínicas Gaspar Vianna, Belém - PA.

2 Centro Universitário do Estado do Pará (CESUPA), Belém - PA.

${ }^{3}$ Universidade Federal do Pará (UFPA), Belém - PA. *E-mail: deivid_ramos45@hotmail.com
} 
embolización arterial. El sureste fue responsable del 48,01\% de los casos. La Región Norte fue responsable del $0,28 \%$. De todos los procedimientos registrados, el 40,63\% son del estado de São Paulo, seguido de Pernambuco $(21,53 \%, n=309)$ y Rio Grande do Sul $(9,69 \%, n=139)$. El Medio Oeste fue responsable de la tasa de mortalidad más alta. Conclusión: Existe una discrepancia en la distribución de casos por estado y región. Se identificó una ausencia total de registros de más de 7 estados durante el período de estudio, lo que indica una falta de esta modalidad terapéutica y / o subregistro y falta de información relacionada con el procedimiento analizado.

Palabras clave: Embolización terapéutica, Hemorragia gastrointestinal, Tracto gastrointestinal.

\section{INTRODUÇÃO}

A Hemorragia Digestiva (HD) é uma emergência médica caracterizada pela perda de sangue originária do trato gastrointestinal e/ou de seus anexos (ALEMA ON, et al., 2012; DALCOMUNE DM, et al., 2019). Essa condição apresenta uma incidência mundial anual de 100 casos a cada 100 mil habitantes, estando associada a altos custos hospitalares por conta da necessidade de internação e elevados índices de morbimortalidade dos indivíduos acometidos (ALEMA ON, et al., 2012; MARTINS AAL, et al., 2019).

Sabe-se que o desfecho desfavorável está diretamente relacionado à falta de acesso aos serviços especializados de assistência médica pública e privada e acredita-se que no Brasil o índice de mortalidade esteja subnotificado, sobretudo em áreas distantes dos centros de referência, pelo distanciamento geográfico dos pacientes em relação aos hospitais, evoluindo a óbito sem um diagnóstico adequado ou tratamento disponível (ALBUQUERQUE MV, et al., 2017; MARTINS AAL, et al., 2019).

A etiologia principal da hemorragia digestiva varia conforme a faixa etária, fatores de risco e aspectos geográficos da população estudada (TARASCONI A, et al., 2019; LIRIO RA, 2016). Destaca-se que em locais mais distantes de grandes centros diagnósticos ou com a indisponibilidade de exames de imagem mais acessíveis para o rápido diagnóstico, os pacientes possuem maior chance de desfecho desfavorável, conforme vários estudos demonstram (DALCOMUNE DM, et al., 2019; NYKÄNEN T, et al., 2018; ŠIRVINSKAS A, et al., 2017).

Os fatores de risco relacionados à maior mortalidade são relacionados à idade avançada, à existência de comorbidades; presença de histórico prévio de melena ou enterorragia, identificação de instabilidade hemodinâmica e taquicardia, além de hemoglobina menor que $8 \mathrm{~g} / \mathrm{dL}$ em exame laboratorial (MARTINS AAL, et al., 2019).

Outra forma, mais acurada, de medir a mortalidade com base na intervenção médica ao utilizar o Escore de Glasgow Blatchford (GBS). Ele aponta o índice de mortalidade e a intervenção médica. Pacientes classificados com GBS maior que 1 possuem alto risco para intervenção médica e mortalidade. No entanto, pacientes com GBS igual a 0 são de baixo risco e podem ter alta precoce com endoscopia ambulatorial posterior (COSTA AF, et al., 2020; MARTINS AAL, et al., 2019).

Em adição ao exposto, a classificação endoscópica de Forrest avalia a probabilidade de recidiva hemorrágica, na presença de estigmas de hemorragia ativa ou recente observando sinais de hemorragia ativa ou recorrentes sob visualização direta durante a EAD (ALEMA ON, et al., 2012; LIRIO RA, 2016). Os pacientes classificados como IA (jorrando), IB (em babação), IIA (vaso visível e sem sangramento) e IIB (coágulo aderente), segundo a classificação de Forrest, englobam o grupo de alto risco de ressangrar (MARTINS AAL, et al., 2019).

Por outro lado, a classificação de Forrest IIC (manchas cobertas de hematina) e Forrest III (camadas limpas das ulcera) são agrupadas como de baixo risco, com chance de novo sangramento de $5-10 \%$ e de 3 a $5 \%$, respectivamente (COSTA AF, et al., 2020; PRETTE-JUNIOR PR, et al., 2018; NYKÄNEN T, et al., 2018).

A HD pode ser classificada em Hemorragia Digestiva Alta (HDA), quando o foco hemorrágico é originário do esôfago, estômago e duodeno ou em Hemorragia Digestiva Baixa (HDB), quando o ponto de origem se situa distalmente ao ângulo de Treitz, ou seja, com origem no intestino delgado, cólon ou reto (COSTA AF, et al., 2020; MARTINS AAL, et al., 2019). 
Entre as etiologias mais comuns da HD, destacam-se a úlcera péptica, varizes esofagogástricas e doença diverticular dos cólons em adultos e idosos (CEA SL, et al., 2019; COSTA AF, et al., 2020; TARASCONI A, et al., 2019; NYKÄNEN T, et al., 2018). Suas manifestações clínicas podem ser diversas e variam desde casos leves/assintomáticos onde é identificada pelo sangue oculto fecal, até casos sintomáticos evidenciados por melena, hematêmese e/ou hematoquezia, as quais podem estar associadas à localização do foco hemorrágico (ALEMA ON, et al., 2012; LIRIO RA, 2016; MARTINS AAL, et al., 2019; MONTEIRO AM, et al., 2021).

Apesar de maior parte dos casos de HD ser autolimitada, mais de $80 \%$ das hemorragias intestinais nãovaricosas cessam espontaneamente. O prognóstico está relacionado principalmente à idade do paciente, à existência de comorbidades, a existência de uso contínuo de medicamentos lesivos à mucosa gástrica e abuso de anticoagulantes. O paciente que chega à urgência com sinais de hipotensão, choque hemodinâmico, coagulopatia tem chance potencial de desfecho desfavorável, culminando no óbito em poucas horas se não tratado adequadamente (MARTINS AAL, et al., 2019; COSTA AF, et al., 2020).

Podendo ainda ter situações mais agudas e/ou graves onde os pacientes evoluem com instabilidade hemodinâmica, onde torna-se necessário o emprego de métodos terapêuticos mais agressivos cuja principal finalidade é controlar o foco do sangramento por meio de medidas mais agressivas, visando a pronta estabilização hemodinâmica do paciente (ALEMA ON, et al., 2012; COSTA AF, et al., 2020; PRETTE-JUNIOR PR, et al., 2018; NYKÄNEN T, et al., 2018).

Nesse contexto, destaca-se o tratamento por embolização arterial, o qual consiste na inserção de um cateter até um vaso próximo à hemorragia para a injeção de bloqueadores do sangramento (DALCOMUNE DM, et al., 2019; MARTINS AAL, et al., 2019). Entre as vantagens desse procedimento, ressalta-se o fato de ser minimamente invasivo e altamente eficaz e entre as desvantagens, destaca-se a necessidade de médico altamente especializado e um serviço com o aparato tecnológico a pronta disposição quando necessário (DALCOMUNE DM, et al., 2019; LAI HY, et al., 2020; NYKÄNEN T, et al., 2018; ŠIRVINSKAS A, et al., 2017).

Sabe-se que esta modalidade terapêutica está disponível no Brasil, por meio do Sistema Único de Saúde (SUS), de maneira gratuita, universal e integralmente para pacientes com quadro de hemorragia digestiva (DALCOMUNE DM, et al., 2019; MARTINS AAL, et al., 2019). Nesse sentido, o objetivo desta pesquisa foi verificar a distribuição desse serviço e a taxa de mortalidade relacionada a embolização arterial de hemorragia digestiva realizados no SUS em todas as regiões e estados brasileiros do período de 2014 a 2020.

\section{MÉTODOS}

Tendo em vista que os dados aqui analisados estão presentes e disponíveis em banco de dados de domínio público, não foi necessário submeter esse estudo ao Comitê de Ética em Pesquisa em Humanos.

Trata-se de uma pesquisa quantitativa, retrospectiva e descritiva, realizada a partir da coletada em base dedados disponibilizada pelo Sistema de Informações Hospitalares do SUS (SIH-SUS/ DATASUS), seguindo a metodologia proposta por SANTOS DR, et al., (2020), sob o código 0406040192, que se refere ao procedimento de embolização arterial de hemorragia digestiva.

Este estudo abrange uma série temporal que vai do ano de 2014 até o ano de 2020, a partir de dados notificados no SIH - SUS por local de internação hospitalar, região, estado, caráter de atendimento, procedimento e ano de notificação.

Foram analisados e incluídos nesta pesquisa todos os casos de pacientes submetidos à embolização arterial de hemorragia digestiva alta/baixa registrados no $\mathrm{SIH}$. Pacientes encontrados fora do período estipulado ou sob tratamento de hemorragia sob outra modalidade foram excluídos da pesquisa. A definição das regiões usadas segue as orientações do Instituto Brasileiro de Geografia e Estatística, base do DATASUS (MINISTÉRIO DA SAÚDE, 2008).

A Taxa de mortalidade em decorrência do procedimento estudado foi calculada pelo próprio DATASUS, utilizando-se do número absoluto de óbitos diretamente relacionados ao procedimento sobre o número absoluto de casos registrados. 
Os dados foram estratificados em tabelas e categorizados por estado, macrorregião, caráter do atendimento e ano. Posteriormente, prosseguiu-se para a análise estatística com o programa BioEstat 5.3, por meio do teste Qui-quadrado, observando o $p$-valor < 0,05 e intervalo de confiança de $95 \%$.

\section{RESULTADOS}

Entre os anos de 2014 a 2020, foram notificados 1.435 procedimentos de embolização arterial no Brasil. Observa-se que a região Sudeste foi responsável por $48,01 \%$ casos $(n=689)$, seguidos do Nordeste $(34,43 \%$, $n=494)$ e Sul $(14,36 \%, n=206)$. O número de casos com a variável região ignorada foi nulo. A Região Norte foi responsável por $0,28 \%(n=4)$. A média de procedimentos registrados por ano foi de 205 casos, sendo o menor valor no ano de 2014 ( $n=45$ casos) e ode maior valor no ano de 2018 ( $n=291)$ (Tabela 1).

Tabela 1 - Distribuição dos casos de embolização de hemorragia digestiva por Região entre os anos de 2014 a 2020 notificados no Brasil.

\begin{tabular}{lccccccccc}
\hline Região & $\mathbf{2 0 1 4}$ & $\mathbf{2 0 1 5}$ & $\mathbf{2 0 1 6}$ & $\mathbf{2 0 1 7}$ & $\mathbf{2 0 1 8}$ & $\mathbf{2 0 1 9}$ & $\mathbf{2 0 2 0}$ & Total & $\%$ \\
\hline Norte & 0 & 0 & 1 & 0 & 0 & 1 & 2 & 4 & 0.28 \\
Nordeste & 21 & 59 & 72 & 112 & 113 & 65 & 52 & 494 & 34.43 \\
Sudeste & 17 & 76 & 109 & 99 & 138 & 122 & 128 & 689 & 48.01 \\
Sul & 4 & 25 & 38 & 18 & 33 & 44 & 44 & 206 & 14.36 \\
Centro-Oeste & 3 & 5 & 4 & 3 & 7 & 13 & 7 & 42 & 2.93 \\
\hline Total & $\mathbf{4 5}$ & $\mathbf{1 6 5}$ & $\mathbf{2 2 4}$ & $\mathbf{2 3 2}$ & $\mathbf{2 9 1}$ & $\mathbf{2 4 5}$ & $\mathbf{2 3 3}$ & $\mathbf{1 4 3 5}$ & $\mathbf{1 0 0}$ \\
\hline
\end{tabular}

Nota: Teste do $x^{2}=<0.0001$. Fonte: Amer ST, et al., 2021; dados extraídos do Sistema de Informações Hospitalares do SUS (SIH/SUS).

De todos os procedimentos registrados, 40,63\% são decorrentes do estado de São Paulo, seguidos de Pernambuco $(21,53 \%, n=309)$ e Rio Grande do Sul $(9,69 \%, n=139)$. Os demais foram responsáveis por 1 a $6 \%$ de todos os procedimentos registrados (Tabela 2 ).

Tabela 2 - Distribuição dos casos de embolização de hemorragia digestiva por estado brasileiro entre os anos de 2014 a 2020 notificados no Brasil.

\begin{tabular}{lccccccccc}
\hline Estado & $\mathbf{2 0 1 4}$ & $\mathbf{2 0 1 5}$ & $\mathbf{2 0 1 6}$ & $\mathbf{2 0 1 7}$ & $\mathbf{2 0 1 8}$ & $\mathbf{2 0 1 9}$ & $\mathbf{2 0 2 0}$ & Total & $\%$ \\
\hline Rondônia & 0 & 0 & 0 & 0 & 0 & 1 & 0 & 1 & 0.07 \\
Amazonas & 0 & 0 & 1 & 0 & 0 & 0 & 2 & 3 & 0.21 \\
Maranhão & 0 & 3 & 2 & 3 & 2 & 1 & 2 & 13 & 0.91 \\
Piauí & 0 & 0 & 1 & 0 & 3 & 4 & 1 & 9 & 0.63 \\
Ceará & 1 & 1 & 2 & 0 & 3 & 3 & 5 & 15 & 1.05 \\
Rio Grande do Norte & 4 & 6 & 6 & 5 & 5 & 6 & 3 & 35 & 2.44 \\
Paraíba & 0 & 0 & 0 & 0 & 0 & 0 & 1 & 1 & 0.07 \\
Pernambuco & 36 & 22 & 38 & 70 & 78 & 38 & 27 & 309 & 21.53 \\
Alagoas & 10 & 13 & 18 & 25 & 10 & 2 & 1 & 79 & 5.51 \\
Sergipe & 1 & 1 & 0 & 0 & 0 & 0 & 0 & 2 & 0.14 \\
Bahia & 8 & 15 & 4 & 9 & 13 & 14 & 12 & 75 & 5.23 \\
Minas Gerais & 7 & 7 & 10 & 7 & 32 & 15 & 15 & 93 & 6.48 \\
Espírito Santo & 0 & 0 & 2 & 1 & 1 & 3 & 1 & 8 & 0.56 \\
Rio de Janeiro & 14 & 15 & 28 & 13 & 7 & 6 & 3 & 86 & 5.99 \\
São Paulo & 84 & 52 & 70 & 77 & 97 & 95 & 108 & 583 & 40.63 \\
Paraná & 5 & 2 & 10 & 6 & 13 & 10 & 9 & 55 & 3.83 \\
Santa Catarina & 4 & 9 & 4 & 2 & 2 & 7 & 6 & 34 & 2.37 \\
Rio Grande do Sul & 15 & 14 & 24 & 11 & 18 & 27 & 30 & 139 & 9.69 \\
Mato Grosso do Sul & 0 & 0 & 1 & 2 & 6 & 3 & 0 & 12 & 0.84 \\
Goiás & 7 & 4 & 0 & 1 & 0 & 2 & 0 & 14 & 0.98 \\
Distrito Federal & 1 & 1 & 3 & 0 & 1 & 8 & 7 & 21 & 1.46 \\
\hline Total & $\mathbf{4 5}$ & $\mathbf{1 6 5}$ & $\mathbf{2 2 4}$ & $\mathbf{2 3 2}$ & $\mathbf{2 9 1}$ & $\mathbf{2 4 5}$ & $\mathbf{2 3 3}$ & $\mathbf{1 4 3 5}$ & $\mathbf{1 0 0}$
\end{tabular}

Fonte: Amer ST, et al., 2021; dados extraídos do Sistema de Informações Hospitalares do SUS (SIH/SUS).

Ressalta-se que os estados do Acre, Amapá, Pará, Paraíba, Roraima, Santa Catarina e Tocantins não tiveram procedimentos registrados de embolização arterial em pacientes com hemorragia digestiva no período de 2014 a 2020 e, por isso, não foram adicionados à Tabela 2. 
Em relação ao caráter de atendimento, $42,6 \%$ ( $n=612)$ foram procedimentos eletivos e $56,9 \%(n=816)$ de urgência. 0,5\% ( $n=07)$ tiveram essa variável classificada como outros (Tabela 3).

Tabela 3 - Distribuição dos casos de embolização de hemorragia digestiva por caráter de atendimento entre os anos de 2014 a 2020 notificados no Brasil.

\begin{tabular}{lccccccccc}
\hline Caráter Atendimento & $\mathbf{2 0 1 4}$ & $\mathbf{2 0 1 5}$ & $\mathbf{2 0 1 6}$ & $\mathbf{2 0 1 7}$ & $\mathbf{2 0 1 8}$ & $\mathbf{2 0 1 9}$ & $\mathbf{2 0 2 0}$ & Total & $\%$ \\
\hline Eletivo & 17 & 67 & 90 & 119 & 156 & 99 & 64 & 612 & 42.6 \\
Urgência & 28 & 96 & 134 & 111 & 134 & 145 & 168 & 816 & 56.9 \\
Outros & 0 & 2 & 0 & 2 & 1 & 1 & 1 & 7 & 0.5 \\
\hline Total & $\mathbf{4 5}$ & $\mathbf{1 6 5}$ & $\mathbf{2 2 4}$ & $\mathbf{2 3 2}$ & $\mathbf{2 9 1}$ & $\mathbf{2 4 5}$ & $\mathbf{2 3 3}$ & $\mathbf{1 4 3 5}$ & $\mathbf{1 0 0}$ \\
\hline
\end{tabular}

Fonte: Amer ST, et al., 2021; dados extraídos do Sistema de Informações Hospitalares do SUS (SIH/SUS).

Ao observar a taxa de mortalidade dos casos de embolização arterial estudados, o Centro-Oeste foi responsável pela maior taxa $(14,29)$, seguido do Sudeste $(11,32)$ e Sul $(10,19)$. Não foram registrados óbitos na região Norte decorrentes de embolização arterial de hemorragia digestiva e, portanto, não foi possível calcular a taxa de mortalidade (Tabela 4).

Tabela 4 - Taxa de mortalidade dos casos de embolização de hemorragia digestiva por Região entre os anos de 2014 a 2020 notificados no Brasil.

\begin{tabular}{lcccccccc}
\hline Região & $\mathbf{2 0 1 4}$ & $\mathbf{2 0 1 5}$ & $\mathbf{2 0 1 6}$ & $\mathbf{2 0 1 7}$ & $\mathbf{2 0 1 8}$ & $\mathbf{2 0 1 9}$ & $\mathbf{2 0 2 0}$ & Total \\
\hline Nordeste & 0 & 6,78 & 2,78 & 2,68 & 4,42 & 6,15 & 9,62 & 4,66 \\
Sudeste & 5,88 & 6,58 & 10,09 & 11,11 & 14,49 & 11,48 & 12,50 & 11,32 \\
Sul & 25,00 & 4,00 & 10,53 & 5,56 & 9,09 & 9,09 & 15,91 & 10,19 \\
Centro-Oeste & 33,33 & 20,00 & 25,00 & 0 & 14,29 & 15,38 & 0 & 14,29 \\
\hline Total & $\mathbf{6 , 6 7}$ & $\mathbf{6 , 6 7}$ & $\mathbf{8 , 0 4}$ & $\mathbf{6 , 4 7}$ & $\mathbf{9 , 9 7}$ & $\mathbf{9 , 8 0}$ & $\mathbf{1 2 , 0 2}$ & $\mathbf{8 , 9 2}$ \\
\hline
\end{tabular}

Fonte: Amer ST, et al., 2021; dados extraídos do Sistema de Informações Hospitalares do SUS (SIH/SUS).

\section{DISCUSSÃO}

A HD é uma condição de elevada incidência que está associada a altos índices de morbidade, mortalidade e custos hospitalares, além de possuir relação direta com o tempo de permanência hospitalar, quanto pior a gravidades maior o tempo de permanência no hospital (ALEMA ON, et al. 2012; DALCOMUNE DM, et al., 2019; MARTINS AAL, et al., 2019; TARASCONI A, et al., 2019). A escolha do tratamento adequado para o quadro de cada paciente é essencial para um bom prognóstico (CEA SL, et al., 2019; COSTA AF, et al., 2020; LAI HY, et al., 2020; NYKÄNEN T, et al., 2018; ŠIRVINSKAS A, et al., 2017).

A realização de anamnese e exame físico minuciosos, complementados com avaliação laboratorial são fundamentais para que o médico realize uma boa avaliação do quadro do paciente e norteie o raciocínio clínico para a busca da etiologia, além de lançar mão de métodos terapêuticos para a estabilização do paciente (COSTA AF, et al., 2020; MARTINS AAL, et al., 2019; NYKÄNEN T, et al., 2018).

Dos 1435 casos notificados no período estudado, verifica-se discrepância na distribuição por regiões, sendo os estados do Sudeste responsáveis pelo maior número de procedimentos realizados, principalmente em relação ao Norte, responsável por apenas 0,28\% (4 casos). Diversos autores afirmam que essa discrepância está relacionada às disparidades econômicas, sociais e estruturais existentes no país (ALBURQUERQUE MV, et al., 2017; MARTINS AAL, et al., 2019; SANTOS DR, et al., 2020).

O Sudeste, além da alta densidade demográfica, é mais desenvolvido que o restante do território brasileiro e, consequentemente, possui maior número de hospitais e profissionais habilitados para a realização desse tratamento imediato (ALBURQUERQUE MV, et al., 2017; ALONSO N, et al., 2017; LAI HY, et al., 2020).

Já a Região Norte, por exemplo, possui número menor de hospitais públicos onde este tratamento é realizado e menor densidade demográfica, tudo isso corrobora para uma menor taxa de tratamento por essa técnica (SANTOS DR, et al., 2020). Sugere-se também a possibilidade de subnotificação e subinformação dos casos, pois uma vez que não há serviços especializados nem cirurgiões vasculares em certas regiões brasileiras, não há acesso dos pacientes ao tratamento e, portanto, não há notificação. Gerando subinformação (DALCOMUNE DM, et al., 2019; REIS JMC, et al., 2021). 
Ao analisar por estado brasileiro, a heterogeneidade torna-se mais evidente, pois enquanto São Paulo e Pernambuco foram responsáveis por mais de $60 \%$ dos procedimentos, outros sete estados não notificaram a realização de nenhum procedimento. Isso se dá, sobretudo, devido à baixa concentração de centros de saúde aptos a realizar a embolização arterial nas localidades não metropolitanas dessas regiões, o que deixa grande parte da população, especialmente a que vive nas áreas rurais, desamparada (ALBURQUERQUE MV, et al., 2017; ALONSO N, et al., 2017; RIBEIRO JM, et al., 2017; STRALEN ACSV, et al., 2017).

Fato descrito por Reis JMC, et al., (2021), que destaca que a maior concentração de cirurgiões vasculares reside em grandes centros urbanos, deixando a população interiorana desassistida. Fato justificado por este estudo pela falta de hospitais equipados nos locais mais distantes para absorver esses profissionais, além da ausência de políticas públicas eficazes para diminuir a concentração profissional em grandes centros (REIS JMC, et al., 2021).

Destaca-se a necessidade de realizar outros estudos que avaliem o itinerário terapêutico/ fluxo assistencial desses pacientes, de modo a estudar o caminho percorrido em direção às capitais brasileiras, para a partir disso, planejar a criação de novos centros de atenção ao paciente que necessite de atendimento médico imediato (DALCOMUNE DM, et al., 2019; OLIVEIRA BLCA, et al., 2019).

Isso evidencia a necessidade de realizar programas em universidades e ações de incentivo à capacitação de médicos para a realização da identificação e tratamento, além de estimular a criação e modernização de hospitais, essencialmente nas regiões Norte e Nordeste, com a finalidade de respeitar os princípios de universalização e descentralização hospitalar (ALONSO N, et al., 2017; DALCOMUNE DM, et al., 2019; OLIVEIRA BLCA, et al., 2019; RIBEIRO JM, et al., 2017; STRALEN ACSV, et al., 2017).

Fazem parte dos principais exames laboratoriais o hemograma completo, bioquímica sérica, testes hepáticos, bilirrubina total e frações, estudos de coagulação, que aliados à uma boa anamnese e exame físicos conseguem subsidiar um bom plano terapêutico objetivando a estabilização do paciente (COSTA AF, et al., 2020; MARTINS AAL, et al., 2019).

Para definir o diagnóstico, pode-se realizar a endoscopia digestiva alta (EDA), a colonoscopia, a cápsula endoscópica, a cintilografia, a angiografia digital e a tomografia computadorizada. A EDA é o método diagnóstico mais sensível e específico nos quadros de Hemorragia Digestiva Alta (cerca de $80 \%$ dos casos de HD) e, por conta disso, é tido como método de eleição e prioritário no manejo do paciente, devendo ser realizado em até 24h (MARTINS AAL, et al., 2019; STRALEN ACSV, et al., 2017).

No que tange ao caráter de atendimento, foi demonstrada tendência de aumento no número de embolizações arteriais no período avaliado, em especial as urgentes. Tal fato pode estar relacionado à melhora da assistência médica de média e alta complexidade bem como um aprimoramento dos sistemas de informação nos grandes centros. Apesar desse resultado positivo, não pode-se afirmar que ele está sendo homogêneo em todo o Brasil, devido às diversas características já mencionadas. (ABOUGERGI MS, 2018; COSTA AF, et al., 2020; LAI HY, et al., 2020; MARTINS AAL, et al., 2019; OLIVEIRA BCLA, et al., 2019).

Em conformidade com o estudo de Covre ER, et al., (2019), nota-se taxas de mortalidade mais elevadas na região Centro-Oeste, em contraste com o Nordeste. Nesse cenário, a mortalidade por hemorragia digestiva em casos de embolização está relacionada ao fato do procedimento ser realizado em quadros de maior complexidade, nos quais há perda de grande volume de sangue ou risco de ressangramento, assim como a presença de fatores de risco como morbidade e história prévia de sangramento gastrointestinal (ABOUGERGI MS, 2018; COSTA AF, et al., 2020; MARTINS AAL, et al., 2019; STAHLSCHMIDT A, et al., 2018).

Para contornar essa situação, o manejo inicial do paciente deve seguir a reposição volêmica adequada, controle do sangramento se utilizando de mecanismos farmacológicos ou endoscópicos, além de controlar potenciais infecções. Para isso, o ideal seria manter o paciente instável em uma Unidade de Terapia Intensiva (UTI), com a realização de medidas de reanimação volêmica, se necessário, com concentrado de hemácias e etc (COSTA AF, et al., 2020; MARTINS AAL, et al., 2019).

Porém, em locais onde não há tais serviços, é possível realizar o manejo inicial com medidas farmacológicas com o objetivo de ganhar tempo até a realização de um tratamento definitivo, sugere-se o 
início de inibidor da bomba de prótons (IBP) endovenoso e fármacos vasoativos, ser for HDA de origem já comprovada, pois é confirmado que tais drogas reduzem o sangramento e a incidência de recidivas (COSTA AF, et al., 2020; MARTINS AAL, et al., 2019).

Embora o presente estudo tenha um vasto panorama do tratamento por embolização arterial da hemorragia digestiva, é necessário destacar como possível limitação a subnotificação e incompletude do SIHSUS (SANTOS DR, et al., 2020a), que deve ser contornada mediante o treinamento dos profissionais da saúde acerca da importância e modo de preenchimento das fichas de notificação, as quais são fomentadas por meio dos formulários de internação do paciente no primeiro dia de internação hospitalar (SANTOS DR, et al., 2020b).

Um dos fatores para essa subnotificação ocorre durante o preenchimento do guia de internação inadequadamente (SANTOS DR, et al., 2020a). O profissional acaba pulando o preenchimento de alguma variável ou simplesmente não preenche informações importantes que servem como base para estudos ecológicos e epidemiológicos (MORAES AP, et al., 2021).

Porém, apesar do exposto, os dados usados para este estudo possuem pequena taxa de incompletude nos sistemas de informação, sendo possível calcular tendências e taxas de frequência e subsidiando o planejamento de ações para melhorar indicadores de saúde em determinadas áreas. Além disso, serve para mostrar as regiões onde a notificação de procedimentos eletivos ou de urgência, relacionados a hemorragia digestiva, precisa ser mais bem trabalhada (MORAES AP, et al., 2021).

Além disso, ressalta-se a escassez de pesquisas que dissertem sobre a epidemiologia dessa condição de modo amplo, visto que a maioria dos trabalhos publicados versa somente acerca da hemorragia digestiva alta (ABOUGERGI MS, 2018; COVRE ER, et al., 2019; STAHLSCHMIDT A, et al., 2018; TARASCONI A, et al., 2019).

\section{CONCLUSÃO}

Observou-se discrepância da distribuição dos casos por estado, região e maior mortalidade na região Centro-Oeste. Foi identificado completa ausência de registros de mais de 7 estados durante o período estudado, apontando inexistência desse tipo de tratamento e/ou subnotificação e subinformação relacionada ao procedimento analisado. Diante disso, torna-se necessária a ampliação de políticas públicas que visem universalizar a opção de tratamento por embolização arterial em casos de hemorragia digestiva, em especial em locais distantes dos grandes centros. Sugere-se a realização de novos estudos investigando o itinerário terapêutico de pacientes eleitos a procedimentos cirúrgicos de urgência que se deslocam às capitais por conta da inexistência em seu município de residência e correlacionar tal variável com a mortalidade.

\section{AGRADECIMENTOS}

À equipe científica agradece grandemente aos residentes e preceptores do Serviço de Cirurgia Geral do Hospital Universitário João de Barros Barreto e Hospital de Urgência e Emergência Metropolitano que dedicaram tempo para orientar este trabalho.

\section{REFERÊNCIAS}

1. ABOUGERGI MS. Epidemiology of Upper Gastrointestinal Hemorrhage in the USA: Is the Bleeding Slowing Down?. Dig. Dis. Sci.. 2018; 63(5): 1091-3.

2. ALBUQUERQUE MV, et al. Desigualdades regionais na saúde: mudanças observadas no Brasil de 2000 a 2016. Cien Saude Colet., 2017; 22(4): 1055-64.

3. ALEMA ON, et al. Endoscopic findings in upper gastrointestinal bleeding patients at Lacor hospital, northernUganda. Afr Health Sci., 2012; 12(4): 518-21.

4. ALONSO N, et al. Surgery in Brazilian Health Care: funding and physician distribution. CBC. 2017; 44(2): 202-7.

5. CEA SL, et al. Incidence of Upper and Lower Gastrointestinal Bleeding in New Users of Low-Dose Aspirin. Clin. Gastroenterol. Hepatol., 2019; 17(5): 887-95-e6. 
6. COSTA AF, et al. Diagnóstico e tratamento da hemorragia digestiva baixa: revisão sistemática. Para Res Med J., 2020; 4: e36.

7. COVRE ER, et al. Tendência de internações e mortalidade por causas cirúrgicas no Brasil, 2008 a 2016. CBC, 2019; 46(1).

8. DALCOMUNE DM, et al. Hemorragia digestiva no estado do Espírito Santo: análise quantitativa e qualitativa dos diagnósticos no hospital estadual de referência. BJHR, 2019; 2(6): 6105-11.

9. LAI HY, et al. Angiography and transcatheter arterial embolization for non-variceal gastrointestinal bleeding. Scand. J. Gastroenterol., 2020; 55(8): 931-40.

10. LIRIO RA. Management of Upper Gastrointestinal Bleeding in Children. Gastrointest. Endosc. Clin. N. Am., 2016; 26(1): 63-73.

11. MARTINS AAL, et al. Hemorragia digestiva alta diagnóstico e tratamento: uma revisão de literatura. Para Res Med J., 2019; 3(2): e07.

12. MINISTÉRIO DA SAÚDE. Pesquisa Nacional de Demografia e Saúde da Criança e da Mulher, 2006. Brasília - DF: Brasil, 2008. Disponível em https://bvsms.saude.gov.br/bvs/pnds/img/relatorio_final_PNDS2006_04julho2008.pdf. Acessado em 21 de março de 2021.

13. MINISTÉRIO DA SAÚDE. Departamento de Informática do SUS (DATASUS). Informações de Saúde. Sistema de informações hospitalares. Brasília - DF: Brasil: 2021. Disponível em: http://www2.datasus.gov.br/DATASUS/index.php?area=02. Acessado em 16 de março de 2021.

14. MONTEIRO AM, et al. Remote ischemic conditioning improves rat brain antioxidant defense in a time-dependent mechanism. Acta Cirúrgica Brasileira, 2021; 36(7): e360707.

15. MORAES AP, et al. Notification of 6442 cases of bone tuberculosis in brazil: epidemiological profile from 2009 to 2018. Coluna/Columna, 2021; 20(2).

16. NYKÄNEN T, et al. Transcatheter Arterial Embolization in Lower Gastrointestinal Bleeding: Ischemia Remains a Concern Even with a Superselective Approach. J Gastrointest Surg., 2018; 22(8): 1394-403.

17. OLIVEIRA BLCA, et al. Evolução, distribuição e expansão dos cursos de medicina no brasil (1808-2018). Trab. educ. Saúde, 2019; 17(1).

18. PRETTE-JUNIOR PR, et al. Tratamento endovascular da hemorragia digestiva aguda por volumoso pseudoaneurisma esplênico: relato de caso e revisão da literatura. J vasc bras., 2018; 17(3): 234-42.

19. REIS JMC, et al. Vascular surgery in the most populous state in the Amazon: socio-professional profile and aspirations of the specialty. Jornal Vascular Brasileiro, 2021; 20(1): e20210039.

20. RIBEIRO JM, et al. Políticas de saúde e lacunas federativas no Brasil: uma análise da capacidade regional de provisão de serviços. Cien Saude Colet., 2017; 22(4): 1031-44.

21. SANTOS DR, et al. Crescimento da vasectomia no Sistema Único de Saúde entre 2009 a 2018: um estudo retrospectivo. Revista Eletrônica Acervo Saúde, 2020a; 12(3): e2822.

22. SANTOS DR, et al. Epidemiology and mortality of thoracolumbosacral spinal arthrodesis in brazil: the last 10 years. Coluna/Columna, 2020b; 19(2): 120-122.

23. ŠIRVINSKAS A, et al. Transcatheter arterial embolization for upper gastrointestinal tract bleeding. Videosurg. Other Miniinvasive Tech., 2017; 4: 385-93.

24. STAHLSCHMIDT A, et al. Predictors of in-hospital mortality in patients undergoing elective surgery in a university hospital: a prospective cohort. Rev. Bras. Anestesiol., 2018; 68(5): 492-498.

25. STRALEN ACSV, et al. Percepção de médicos sobre fatores de atração e fixação em áreas remotas e desassistidas: rotas da escassez. Physis., 2017; 27(1): 147-72.

26. TARASCONI A, et al. Transcatheter arterial embolization versus surgery for refractory non-variceal upper gastrointestinal bleeding: a meta-analysis. World J Emerg Surg., 2019; 14(1). 